\title{
Dual Axis Tracker for Photovoltaic Panel
}

\author{
Priyabrata Pattanaik
}

\begin{abstract}
Main challenge of solar-tracking systems are the sunlight detection, providing position and delay of $P V$ movement, designing control module for low consumption dc servo motor(s). Objective of this paper is designing and implementing automatic control for detecting maximum solar light to a solar panel. The two-axis solar tracker is used for optimizing conversion of solar energy into electrical energy, at cost of the mechanical and the maintenance need, for the best efficiency. The hardware development, two dc servomotors are adjusted which is controlled by drive module moving panel by using four Light-Dependent Resistor (LDR) to provide the analog signals; the signals are processed by ATMEGA328P micro-controller with Arduino.
\end{abstract}

Keywords: Reduced Instruction Set Computer, Azimuthelevation (AE), dual-axis solar tracker, microcontroller

\section{INTRODUCTION}

Renewable energies have been viewed as a great source for the past two decade, and are currently widely-used in industrial and household applications [1]. Energy is key reason for enhancing and increasing the development and growth of any country. The per capita energy consumption is considered as index of the prosperity of the nation. With the increasingly high alarming rate of depletion of nonrenewable sources of energy, renewable energy [2]sources such as wind energy, hydro energy, solar energy etc. does exists in large abundance in our nature and environment [3]. All this non-conventional energy is ready to be extract from the environment and most importantly it is a cleaner source of energy in comparison with non-renewable source of energy.

Since non-renewable source of energy is decreasing in large amount the renewable source of energy are getting huge attention for future generation of energy.

This paper involves design and proper control of prototype of the dual axis solar-tracking system for solar panel to improve the energy efficiency. The tracking system for solar panel improves the harnessing of maximum sun's energy [4]. The system comprises of two parts: mechanical unit and electrical unit. The electric unit consists of four identical LDR(s) (Light Dependent Resistor) as an input, ATMEGA microcontroller is used as a controller, and two servomotors are used as an output.

\section{METHODOLOGY RESULTS}

The project involves design of a dual-axis solar tracker for solar panel, movement given by dc geared servomotors, and analyzing its working and performance. It comprises mainly three components: Four LDR(s) for sending input signal, an Arduino Uno with ATMEGA328P microcontroller and two dc servomotors for motion of panels. The analog signals from LDR(s) are transmitted to the ATMEGA328P, the ATMEGA328P converts the analog signal into digital signals by using Analog to Digital converters, and the signal is sent to twin PWM controlled dc servomotors to move solar panel towards sun light. The whole system design is categorize into two different parts mechanical and electrical part.

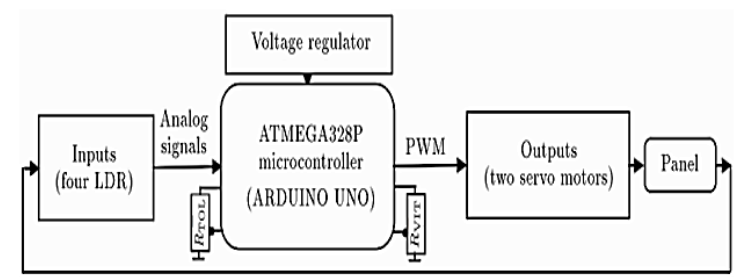

Figure 1

Different components of the electric design:

This section concerns the components of the solar tracker to orient the panel towards solar rays. The components of the hardware design are four LDRs, an Arduino Uno microcontroller and two de servomotors.

\section{Photo-resistor:}

Light-Dependent Resistor (LDR), as shown in the Figure 2, a LDR is made out of a semiconductor having high resistivity and is an electronic component whose resistance value decreases as the sun light intensity increases, as shown in the Figure 3. This intensity of light sensed by the LDR is used as an analog input voltage for ATMEGA microcontroller. For our application, four identical LDRs are used in a sight glass disposed on panel surface as illustrated in Figure 4. Sighting glasses composed of four LDR optically isolated by an opaque plate, as shown in Figure 5(c); thus, their illumination marks can be similar only if the sight glass is pointed toward the sun. This sight glass is fixed on a PV panel and placed on the same plan, forming a sensor designed to detect the sun position. A signal error is generated if system is non- pointed. It is the signal that will be used by microcontroller to deliver the adequate control to dc servomotors. To process this signal, a voltage divider has been used for each LDR. 

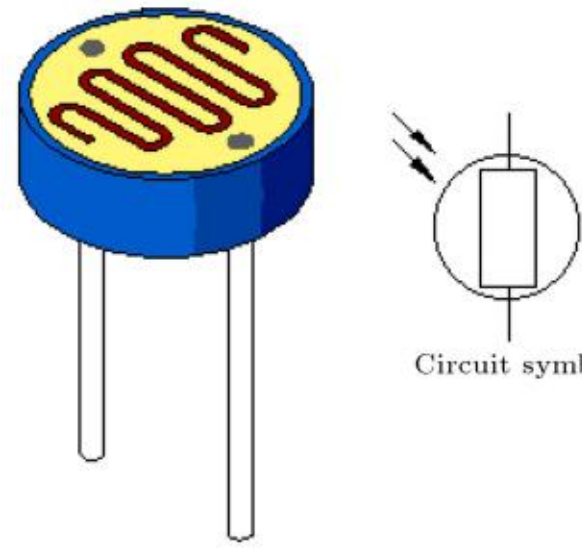

Circuit symbol

Figure 2

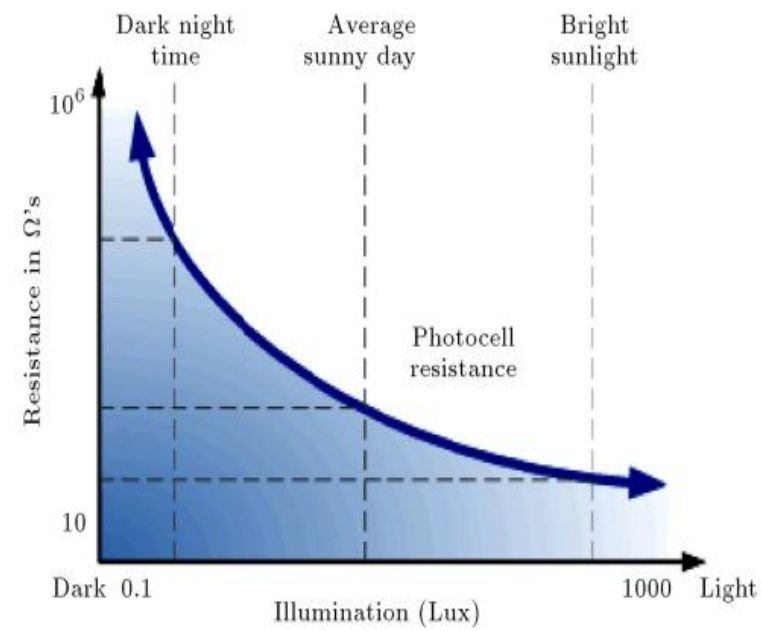

Figure 3

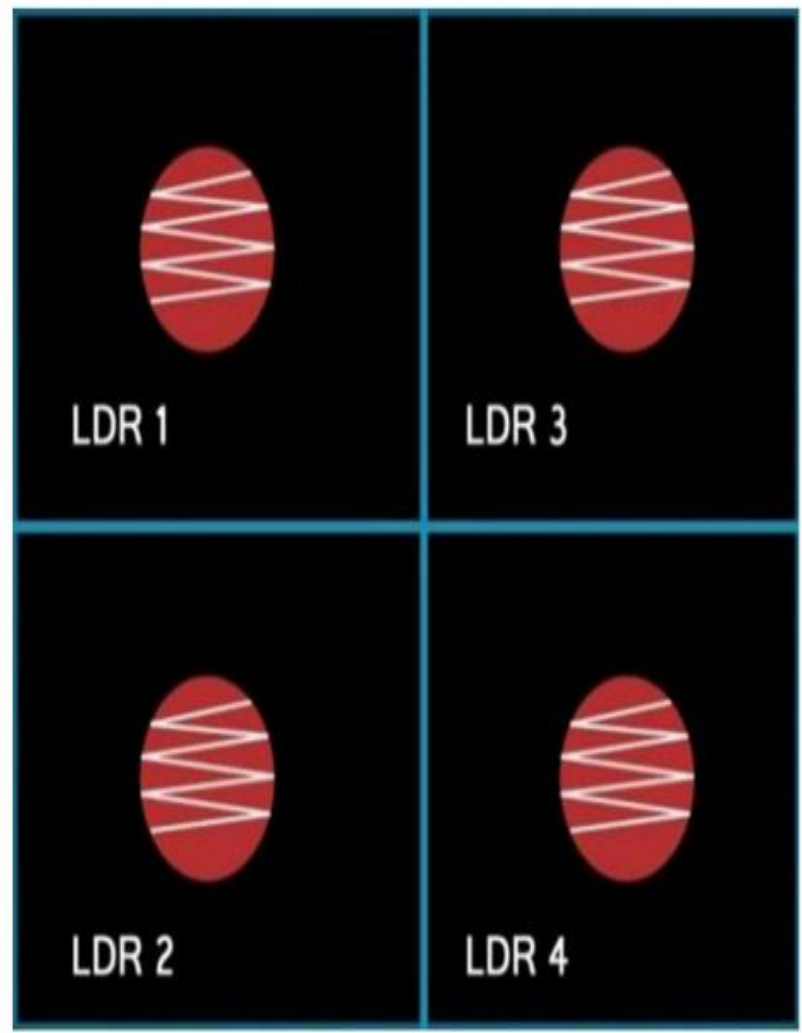

Figure 4
The output voltage Vout is proportional to light intensity. Vout will increase during maximum light is captured by LDR, and the value of Vout is weak during a shadow on a LDR, expressed by:

$$
V_{\text {out }}=\frac{V_{\text {in }} \cdot R}{\left(R+R_{\mathrm{LDR}}\right)} .
$$

\section{Servomotors:}

Objective of servomotors is providing an exact movement as a response to an external control. It is an actuator which mixes electronics, mechanics, and automatics. Servomotors as shown in Figure 5 high torque with very large power range is available in a wide variety of frame sizes from small to large; it is capable of running huge machines, has an excellent power capacity to weight size ratio given their best efficiency (80-90\%), and also has low speed applications given with low friction and correct gear ratio leading to a very low heat production, vibration, and very little noise. The servomotor in Figure 6 is an assembly of three basic blocks: a body comprising all the mechanics and electronics, a cable to lead power and the control (reference signal), and a pioneer attached to the servomotor's axis. Herein, the pioneer is attached to the mechanical parts to move (arm, wheel, etc.). This servomotor type requires regulated supply voltage of $5 \mathrm{~V}$. It comprises of 3 wires: ground, positive, and signal wires.

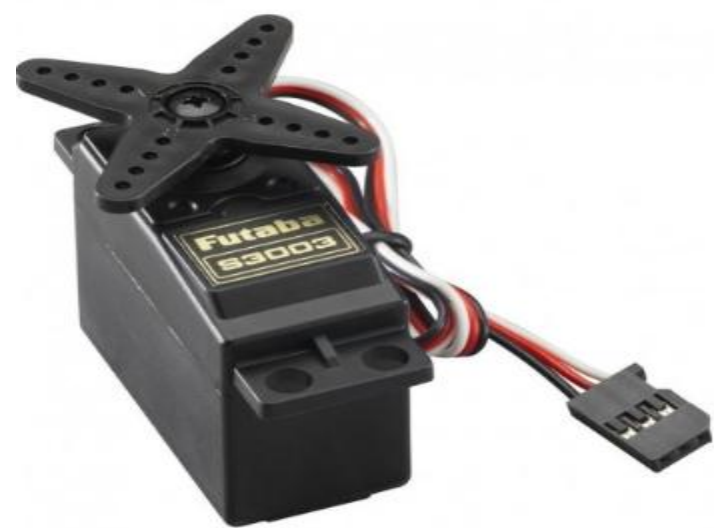

Figure 5

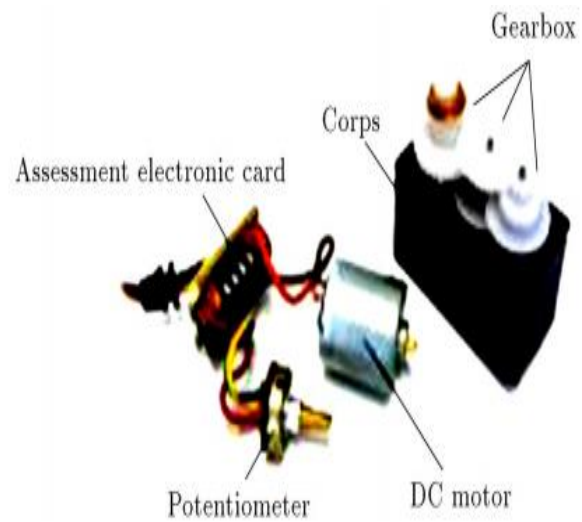

Figure 6

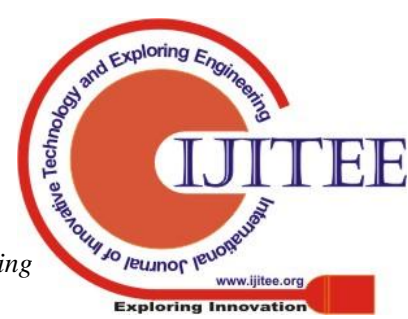


The servomotor used in this project is an assembly of four parts: an electrical dc motor, a gearbox, a position-sensing device which is usually a potentiometer, and an electronic card to control and assess the motor. The servomotor body is composed of an electronic card receiving the reference PWM as shown in Figure 8 signal to do the assessment. This card controls dc electrical motor that will drive the pioneer through the speed-reducing gear, as illustrated in Figure 7.

The gearbox has two functions:

- Reducing the speed to provide an accurate tracking of relative position of the servomotor and avoiding any system damage when movement is driven by its speed if it is very high;

- Increasing the torque by its mechanical movement of rotation. At an instant $t$, if we decrease speed leaving the same mechanical power Pmec, this will increase torque T.

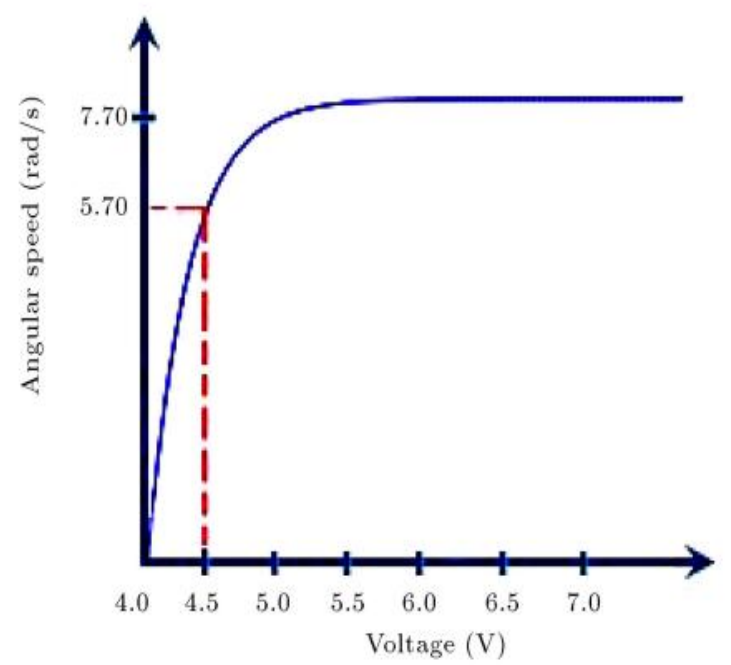

Figure 7
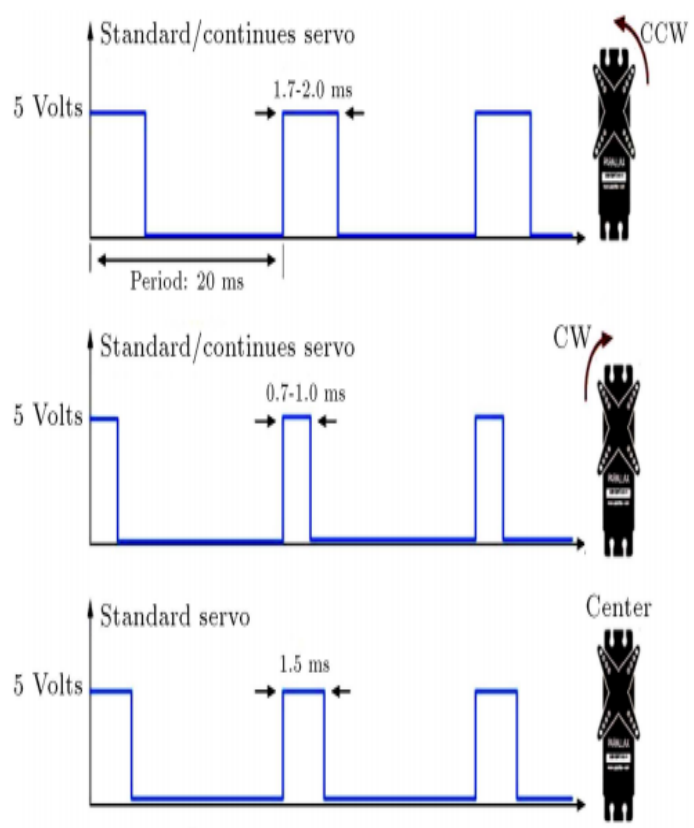

Servo motor PWM timing diagram

Figure 8

Microcontroller and programming:

Our active sun tracker is controlled by PC program using an Arduino Uno. This section presents the specifications of the microcontroller and the developed programming to conduct the exact system working.

The use of microcontrollers for programming circuits comes with various strong and real advantages. In fact, we have found the spectacular evolution of IC (Integrated Circuit) design in recent years. It simplifies tracing of the printed circuits to data/address bus, increasing system reliability, and reducing cost. The microcontroller converts the analog signals captured from the LDR to the digital signal that will be compared to the tolerance input signal, to be transmitted to servomotors in order to move the PV panels where it can receive the maximum amount of sunlight.

The choice of the microcontroller is done on the basis of its application: its preference to fix a family. Even though the PIC microcontroller of Microchip has contributed much to popularize the architecture Reduced Instruction Set Computer (RISC) and a great deal of information in the world of microcontrollers and in the related literature has been devoted to informatics architectures, they are not the only circuits in the market. The microcontrollers of AVR family from Atmel, to which this paper is dedicated, use this architecture and, hence, benefit from various advantages.

The AVR family has many advantages: inexpensive, lowenergy consumption, and a good support multi-platform. They are successful, given the following specifications:

(a) Easy $\mathrm{C}++$ programming for most basic functions;

(b) Low cost and large availability; and

(c) Low-energy consumption.

Figure 9 shows synopsis of internal hardware architecture of ATMEGA328P microcontroller. 


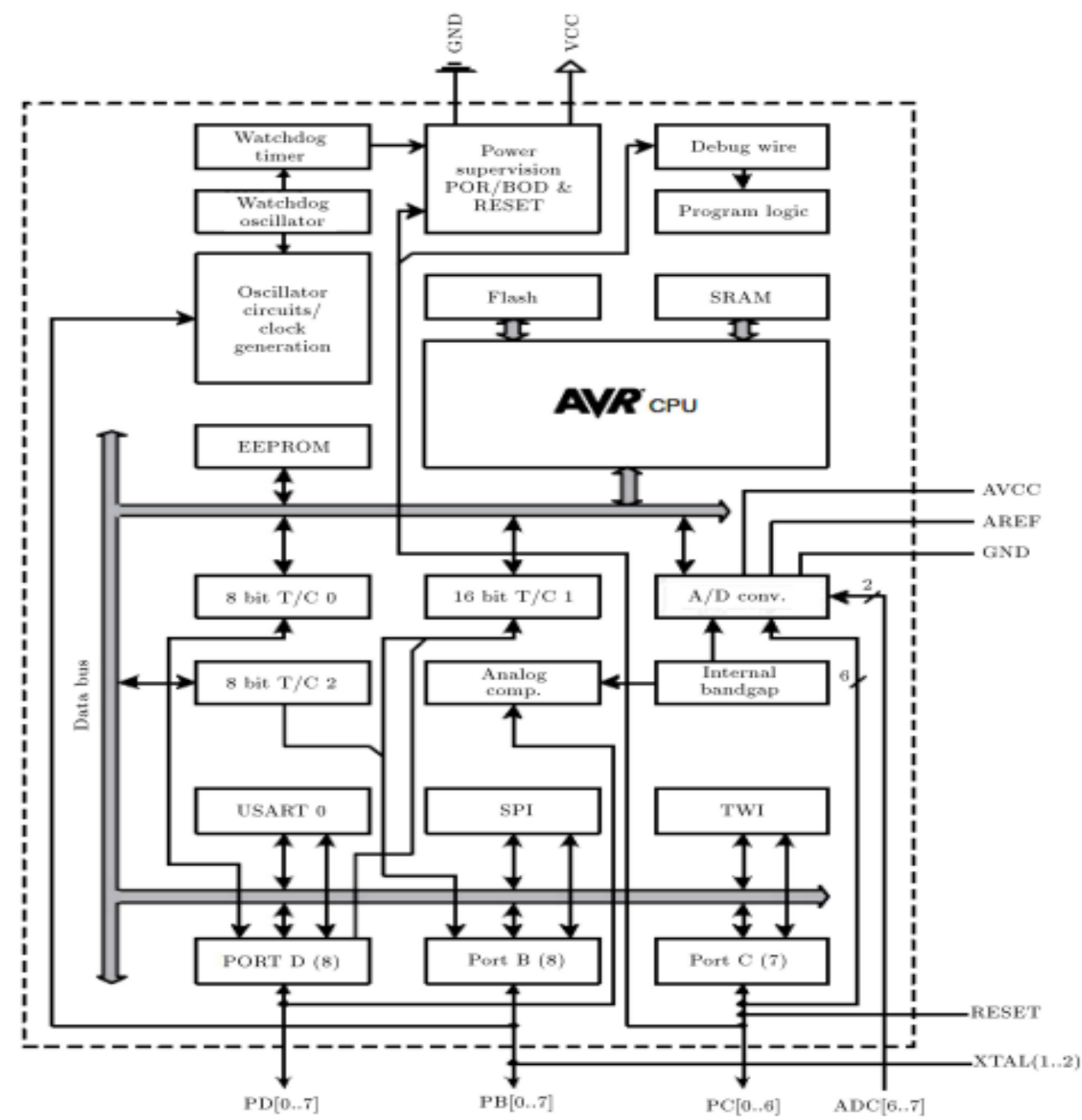

Figure 9

\section{Design of the solar tracker control circuit:}

Dual axis solar tracker works on four LDR(s) was constructed and tested to set optimal values of the two potentiometers RVIT and RTOL, shown in Figure 10. The RTOL potentiometer tolerance calculates the angle between upside couple of (LDR1 and LDR3) and downside couple of the (LDR2 and LDR4) sensor. After some trial error testing, optimal angle between all photo resistors was calculated experimentally to set tolerance voltage TOL. Approximate result was found because system was working in close loop system that is conventional control method of the sun tracking system by photo resistors. The photo sensor(s) used for discriminating sun's position and then send proper analog electric signals, converted to digital signals, proportional to the controller error, that actuates motors to track sun light [5][6]. Circuit scheme of designed system for horizontal and vertical axes is shown in the Figure 10. When sun moves to east or to west, either couple (LDR3 and LDR4) or (LDR1 and LDR2) will going to receive more sun light, transforming collected solar light illumination to an electrical voltage VLDR 3, VLDR 4 or VLDR 1, VLDR 2 using voltage dividers (Equation (1)). After that, they are sent to the ADC ports for angle of altitude and for azimuth's angle tracking to microcontroller to make comparison with tolerance of the input signal value TOL sent to ADC port to control the servomotors. These ports performs orientation of PV panel towards sun light. Rotation direction for azimuth and altitude angle tracking the respective horizontal $(\mathrm{H})$ and vertical (V) motors are controlled by using dual differential amplifier. 


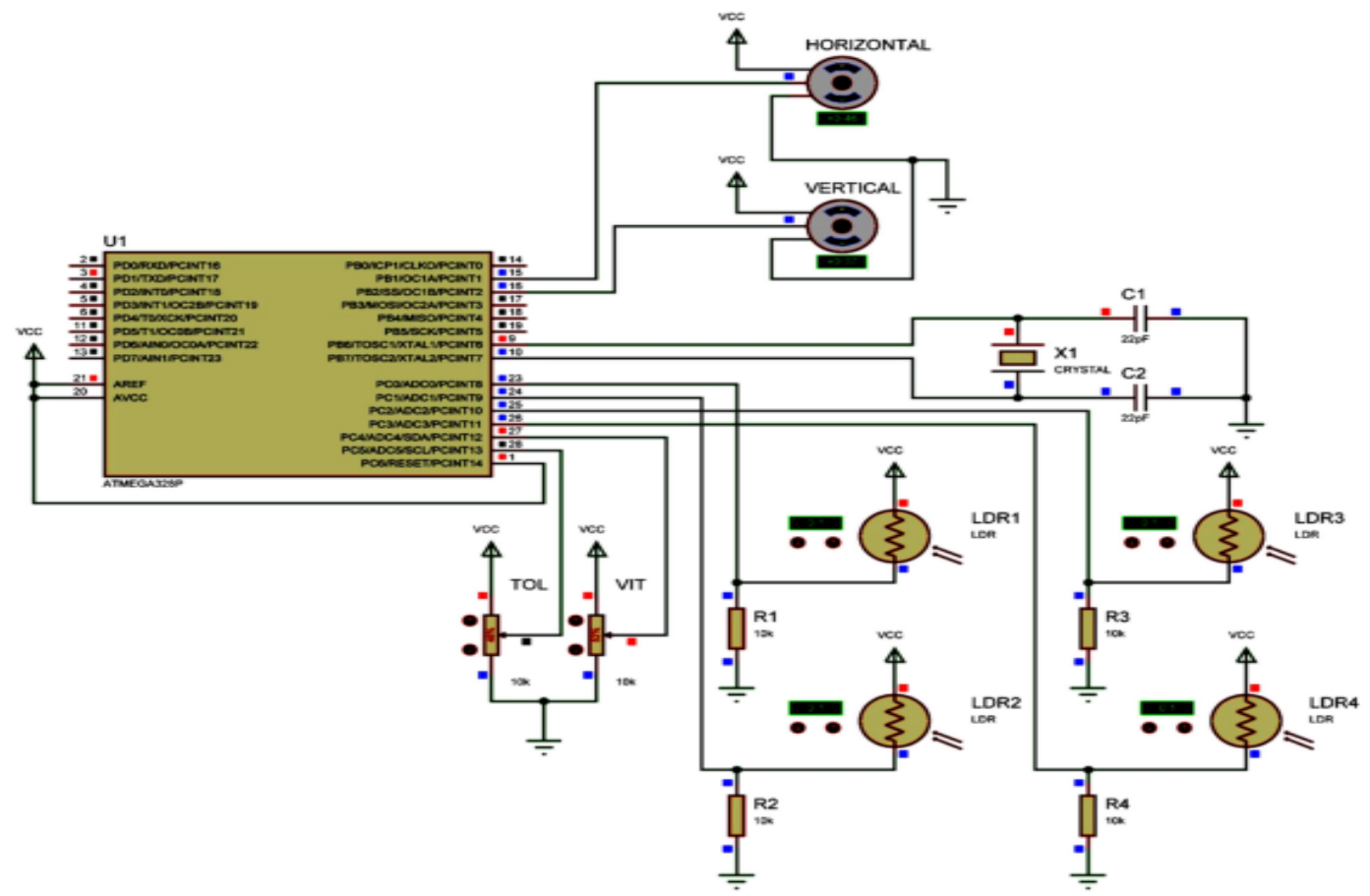

Figure 10

When servo motors rotate, $\mathrm{H}$ and $\mathrm{V}$ axis changes positions which will modify potentiometers' resistance vale mounted on shafts and provides $\mathrm{H}$ and $\mathrm{V}$ value with a feedback voltage. They will be then compared to the PWM control signal to determine position of the de servo motors. Finally both $\mathrm{H}$-motor and $\mathrm{V}$-motor are turned in direction as clockwise (cw) and counter-clockwise (ccw) that absolute value of dHoriz and dVert becomes less than TOL, and dc motors then stops.

\section{CONCLUSION}

Described prototype satisfies control conditions for decent working of system; hence, the results are encouraged. In fact, program repartition allows to distinguish the various realized operation. Instruction were followed from program guidelines in methodical repairing of function. It facilitates modifications that could be taken during these works. The total cost is very low, and period of operation is about 45 days. It has effective contribution to environment and also could be improved. The two degrees of freedom were made to track sun position. In this case, system was tested by using a torch light to shine at the sensors; constructed tracker followed it around, which was a successful in verifying its correct working. Micro-controller was used to control the exact position of dc servomotors that ensures the point to point stable movement. Microcontroller was designed to rotate panel from 0 degree to 180 degree. Presented dual axis PV panel tracking system provides the solar panel perpendicular to sun throughout year to improve efficiency of system. As advancements made in solar trackers technology have decreased investment prices, the project could be extended to power supply for isolated villages or farms by mounting highly optimal.

\section{REFERENCES}

1. M. Abdollahpour, M. R. Golzarian, A. Rohani, and H. Abootorabi Zarchi, "Development of a machine vision dual-axis solar tracking system," Sol. Energy, 2018.

2. V. K, "Designing a Dual Axis Solar Tracking System for Maximum Power," J. Electr. Electron. Syst., 2016.

3. I. Sefa, M. Demirtas, and I. Çolak, "Application of oneaxis sun tracking system," Energy Convers. Manag., 2009.

4. K. K. Chong et al., "Prototype of Dense-array Concentrator Photovoltaic System Using Non-imaging Dish Concentrators and Cross Compound Parabolic Concentrator," in Energy Procedia, 2017.

5. H. Bentaher, H. Kaich, N. Ayadi, M. Ben Hmouda, A. Maalej, and U. Lemmer, "A simple tracking system to monitor solar PV panels," Energy Convers. Manag., 2014.

6. S. Yilmaz, H. Riza Ozcalik, O. Dogmus, F. Dincer, O. Akgol, and M. Karaaslan, "Design of two axes sun tracking controller with analytically solar radiation calculations," Renewable and Sustainable Energy Reviews. 2015. 